\title{
Hidratação pré-ovulatória em peixes: Um caráter de oriyem marinha? ${ }^{1}$
}

\author{
Paulo de Tarso da Cunha Chaves ${ }^{2}$
}

\begin{abstract}
Based on original and literature data, this study discusses the close relationship that seems to exist between the pre-ovulatory hydratation $(\mathrm{POH})$ on fish oocytes and the marine environment, contrasting with the absence of this process on freshwater species. The difference is mainly explained by the necessity or not of intraoocitary water on eggs flutuation, in one or another situation, according to the surroundings. Once accepted this hipothesis, it is proposed that POH should be an efficient indicator of the adaptation to freshwater environment in fish species originated from marine groups.
\end{abstract}

\section{INTRODUÇÃO}

A hidratação pré-ovulatória sofrida pelos ovócitos dos peixes (HPO), entre o término da vitelogênese e sua eliminação ao meio, tem origem na transferência de líquido da circulação sangüínea da fêmea até o gameta em desenvolvimento, no qual promove coalescência dos grânulos de vitelo e substancial aumento de volume. A necessidade de ocorrência do processo, ao menos as espécies marinhas com ovos pelágicos, reside na aquisição de maiores facilidades à flutuação dos ovos, já que o citoplasma hidratado é menos denso que a água do mar (GINZBURG, 1968). Todavia, tal redução de densidade não se mostra suficiente para permitir a flutuação dos ovos em espécies de água doce, meio cuja densidade é por sua vez inferior à do citoplasma hidratado. Neste ambiente, segundo CRAIK \& HARVEY (1987), a baixa densidade dos ovos é garantida principalmente pelo seu teor em lipídeos, permitindo supor-se que a HPO nos teleósteos dulceaqüícolas seja até mesmo dispensável, ocorrendo com intensidade ou freqüência menor que nos teleósteos marinhos.

De fato, os registros de HPO nos teleósteos não são constantes, sendo omitidos para um grande número de espécies. Face a isso, baseando-se na suposição teórica acima exposta, executou-se neste trabalho uma comparação entre os registros de HPO em espécies marinhas e dulcea-

1 Parte de Tese apresentada ao Instituto Oceanogråfico da Universidade São Paulo. Trabalho parcialmente financiado pela FAPESP e pela CIRM.

2 Programa de Pós-graduação, IOUSP - C.P. 9075, CEP 05508 São Paulo, SP. 
qüícolas, objetivando detectar e explicar a presumida diferença no grau de manifestação do processo em cada um desses ambientes. Caso a diferença efetivamente exista, será possível classificar-se a HPO como sendo um caráter ovariano fortemente relacionado ao meio, indicador em potencial do grau de adaptação à água doce pelas espécies pertencentes a grupos originalmente marinhos.

\section{MATERIAL E MÉTODOS}

Uma tabela reunindo os registros ou não de HPO foi elaborada a partir da compilação de dados originais e de literatura referentes a 43 espécies, distribuídas em 5 ordens. Para a escolha desses grupos, teve-se o critério de explorar diferentes graus evolutivos e de representatividade ambiental, conforme segue:

- Clupeiformes, de representatividade marinha e dulceaqüícola;

- Characiformes, exclusivamente dulceaqüícolas;

- Siluriformes, principalmente dulceaqüícolas;

- Gadiformes, eminentemente marinhos; e

- Perciformes, de representatividade marinha e dulceaqüícola.

Esta compilação não foi aleatória, pois se por um lado todas as espécies dulceaqüícolas encontradas na pesquisa acham-se aí referidas, o mesmo não acontece para as espécies marinhas, dentre as quais só serâo mencionadas aquelas em que a hidratação foi histologicamente registrada. Portanto, não se tecerá considerações sobre o grau de manifestação do processo no meio marinho, mas apenas sobre os registros diferenciados num ambiente ou no outro.

Para ilustrar a HPO, apresentam-se também fotografias de preparados histológicos confeccionados pela técnica de rotina para coloração Hematoxilina-Eosina, precedida de fixação em formol a $10 \%$. Este material, a exemplo daquele que resultou nos demais dados originais a seguir apresentados, foi coletado em águas do litoral norte do estado de São Paulo.

Em 5 das espécies efetuaram-se medições de ovócitos ao longo das etapas de vitelogênese e hidratação. Os volumes foram estimados mediante aplicação da fórmula da esfera, a partir do diâmetro vertical mediano de ovócitos examinados ao microscópio estereoscópico, dissociados de gônodas já identificadas histologicamente.

\section{RESULTADOS E DISCŨSĀO}

Os dados reunidos à Tabela I demonstram que, realmente, a ocorrência de HPO nos ovócitos de teleósteos possui uma relação muito mais estreita com o ambiente marinho que com o dulceaqüícola. Das espécies desovantes neste meio, nenhuma das aqui compiladas acusou a existência do caráter, sendo isto verdadeiro não apenas para aquelas pertencentes a grupos primariamente dulceaqüícolas (Characiformes, Siluriformes), mas também para as derivadas de grupos originalmente marinhos (Pellona 
Vol. 6(3), 1989

castelnaena, Família Cichlidae, Plagioscion squamosissimus, Pachipops trifiris). Já entre as espécies marinhas, inclusive algumas pertencente às mesmas famílias das anteriormente referidas, os registros de HPO são bastante comuns, sugerindo que para a ocorrência do processo, mais que fatores inerentes ao grupo sistemático ou posição evolutiva, o elemento decisivo deve mesmo ser o ambiente.

TABELA I - Tabulação de informações sobre registro ou não de HPO, em 43 espécies, por ambiente e segundo o grupo sistemático.

ES PÉCIE

\section{CLUPEIFORMES}

Engraulis mordax

Opisthonema libertate

I lisha afrioana

Sardinella brasiliensis

Harengula clupeola

Clupea harengus

Sardinops sagax sagax

Pellona castehnaena

CHARACIFORMES

Brycon cephalus

Chalceus macrolepidotus

Hoplias malabaricus

Semaprochilodus spp

Serrasalmus spp

SILURIFORMES

Tandanus tandanus

Pimelodus maculatus

Rhamdia sapo

Rhinelepis aspera

Plecostomus commersonii

GADIFORMES

Melanogrammus aeglefinus

Urophycis brasiliensis

Merluccius merluccius

M. productus

M. gayi peruanus

PERCIFORMES

Famf́lia Cichlidae

Geophagus brasiliensis

G.surinamensis

Astronotus ocellatus

Cichla ocellaris

Tilapia nilotica

\section{AMBIENTE \\ REFERÊNCIA \\ mar å.d.} TORRES-VILLEGAS et al., 1985

$+$

$+$

$+$

$+$

$$
+
$$$$
+
$$

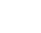$$
-\mathrm{CH}
$$

- CHAVES \& VAZZOLER, 1984

- CHAVES, 1985

- DAVIS, 1977

- GODINHO et al ., 1974

- CUSSAC \& MAGGESE, 1986

- AGOSTINHO, 1985

- AGOSTINHO'et al., 1982

$+\quad$ ROBB, 1982

$+\quad$ dados originais

+ $\quad$ ANDREU, 1956

+ $\quad$ FOUCHER \& BEAMISH, 1980

+ $\quad$ ANDRIANOV \& LISOVENKO, 1983

- BARBIERI et alo, 1981

- CHAVES, 1985

- CHAVES, 1985

- CHAVES, 1985

- BABIKER \& IBRAHIM, 1979 
Revta bras. Zool.

T. mossambica

Outras famflias

Trachurus trachurus

Liza aurata

Eucinostomus argenteus

Lopholatilus chamaeleonticeps

Dules auriga

Pagrus major

Plagioscion squamosissimus

Pachipops trifiris

Isopisthus parvipinnis

Menticirrhus americanus

Cynoscion striatus

Stellifer brasiliensis

Seriphus politus

Umbrina canosai

- ARAVIDAN \& PADMANABHAN, 1972

$+$

$+$

$+$

$+$

$+$

$+$

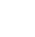

+ : registro positivo

- : registro negativo
MACER, 1974

KULIKOVA \& LOSHAKOVA, 1982

dados originais

ERICKSON et al., 1985

dados originais

MATSUYAMA et al., 1987

- CHAVES, 1985

- CHAVES, 1985

dados originais

CASTILLO, 1986; dados originais

dados originais

dados originais

DEMARTINI \& FOUNTAIN, 1981

HAIMOVICI, 1982

mar: especie marinha

åd o: espécie dulceaqǘfcola

Uma correlação de tal nível conduz assim para um interessante raciocínio de convergência adaptativa, uma hipótese segundo a qual as espécies primariamente dulceaqüícolas nunca teriam adquirido o caråter, enquanto os grupos secundariamente dulceaqüícolas o teriam perdido, em algum estágio de sua adaptação à água doce. Esta conclusão acompanha-se de um bom fundamento teórico, pois mesmo que a hidratação eventualmente ocorra também nos ovócitos de espécies dulceaqüícolas (por outras necessidades que não a de diminuir sua densidade), é permissível que nestas o processo se dê não antes da ovulação e desova, mas sim após. Em água doce isto é viável, pois em sendo o citoplasma intra-ovocitário isosmótico aos fluidos corporais da fêmea (HOLLIDAY, 1969, apud BOND, 1979) (fato que o torna hiperosmótico em relação ao meio), e tendo-se em conta que os ovos não-fertilizados ainda são desprovidos de capacidade osmorregulatória (HOLLIDAY, op. cit.), existe uma tendência natural de os ovócitos após a desova se hidratarem passivamente. Para as espécies marinhas isto é muito improvável, uma vez que o citoplasma intra-ovocitário é hiposmótico ao meio, condição que não favorece o ganho de água, mas apenas a perda.

Entretanto, que proveito teriam as espécies dulceaqüícolas em não hidratar seus ovócitos antes da desova? Uma ausência de HPO teoricamente apresenta diversas vantagens para as espécies que dela se abstêm, à medida em que a ocorrência do processo implica encargos como os seguintes:

$1^{\circ}$ ) Um certo dispêndio energético para a fêmea, pela transferência de líquido da circulação. A Fig. 1 ilustra a seqüência do processo de hidrataçẩo em algumas espécies marinhas, evidenciando o grande volume de líquido que os ovócitos recebem após o término da vitelogênese. 

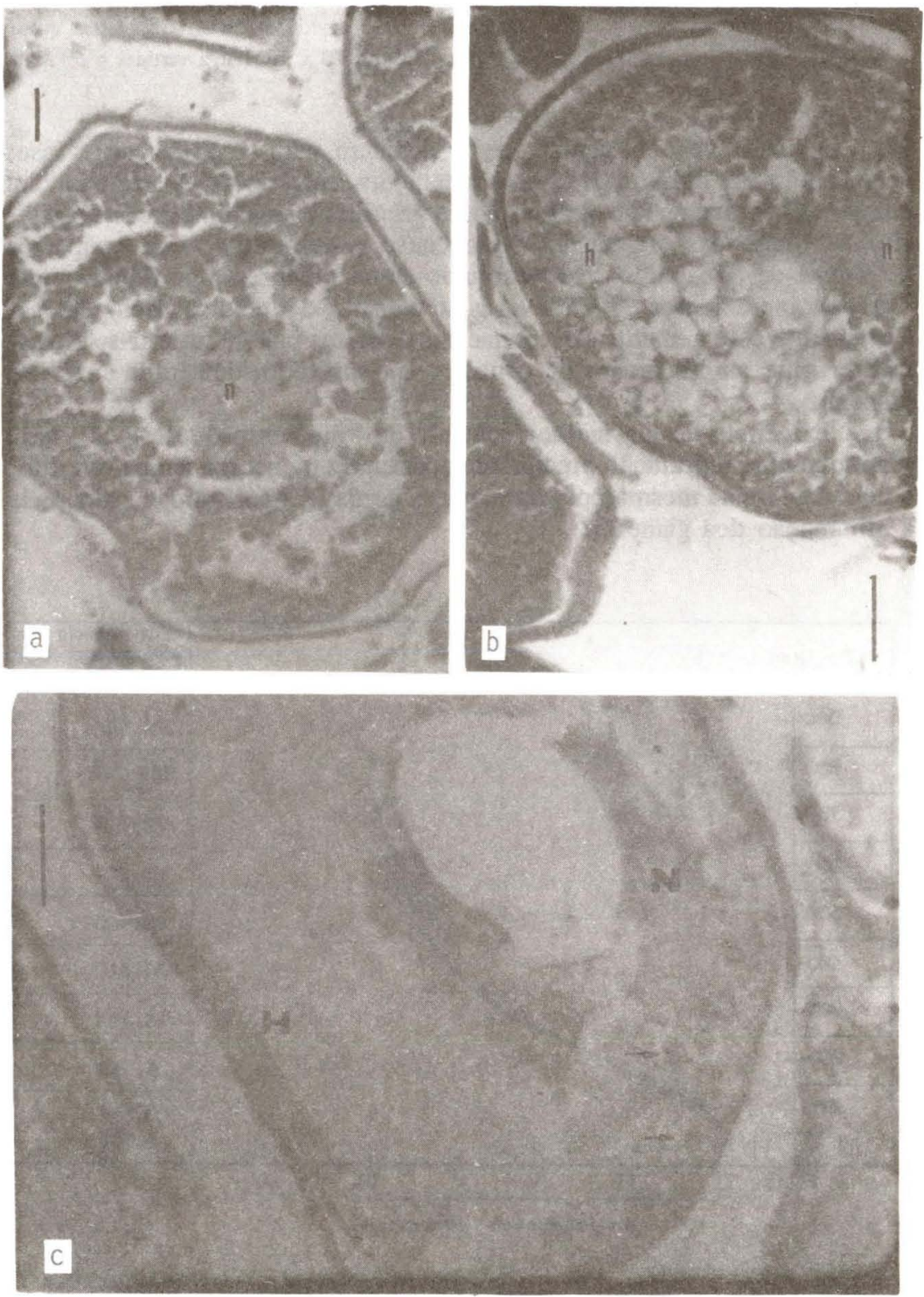

FIG. 1 - Seqüência do processo de hidrataçảo pré-ovulatória teleósteos marinhos. a: folículo ovariano maduro de Ctenoscialna gracilicirrhus, com vitelogênese completa e núcleo ainda no centro do ovócito (n). b: folf́culo ovariano em iń́cio de hidratação de Dules auriga. Observase que o núcleo (n) deslocase para um dos polos do ovócito, aquele que mantém gotfculas de vitelo. No pólo oposto, citoplasma em coalescência 
(h). c: folículo hidratado de Menticirrhus americanus. Núcleo (N) situado num dos polos do ovઠ́cito. Poucos grânulos de vitelo acidofilo (setas). Citoplasma quase totalmente coalescido $(\mathrm{H})$. Coloração $\mathrm{HE}$, aumento $10 \times 10$. Barra vertical $=50 \mu \mathrm{m}$.

2:) Um maior peso e volume a ser ocupado pelos ovários nesta fase, acarretando ao indivíduo severas alterações estruturais, senão também comportamentais. A Fig. 2 apresenta a progressão das dimensões ovocitárias ao longo das etapas de vitelogênese e hidratação, em 5 espécies do litoral de São Paulo, demonstrando como o ingresso de líquido pode provocar incrementos de 6 a 8 vezes no tamanho das células vitelogênicas. Certamente este aumento de volume dos ovócitos poderá refletir-se diretamente sobre as dimensōes da gônada como um todo, dentro da cavidade visceral.

3) A exigência de um melhor controle do "momento de desova", haja visto que uma hidratação precipitada acentuaria o encargo acima referido, e uma demora na mesma poderia implicar perda de oportunidade adequada à eliminação dos gametas.

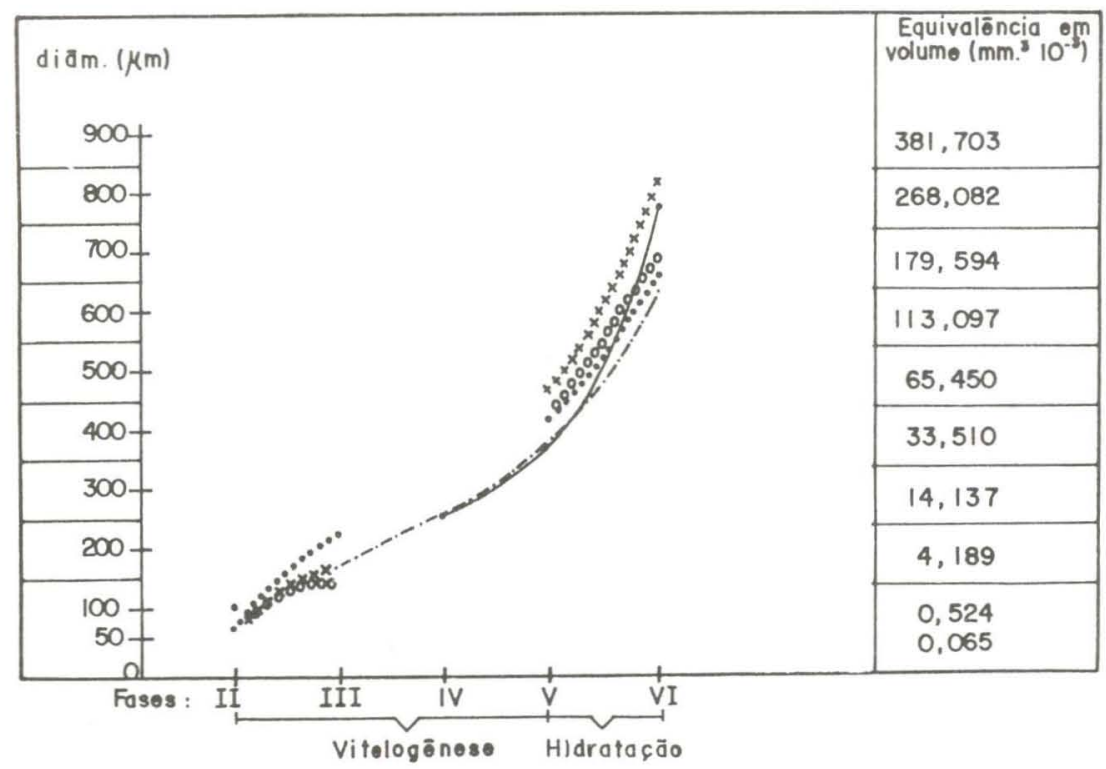

Harengula clupeola, ...... Urophycis brasiliensis, -.- Eucinosfomus
argenteus, $\times \times \times \times$ Isopisthus parvipinnis, 00000 Menticirrhus americanus.

FIG. 2 - Representação gráfica do aumento de volume ovocitário, em função do diâmetro estimado de cada fase, para 5 espécies de teleósteos do litoral de Sảo Paulo. Verifica-se que o incremento de volume sofrido pelo ovócito, quando do processo de hidrataçäo, pode ser da ordem de 6 a 8 vezes. 
Assim, pode-se concluir que para as espécies de grupos primariamente dulceaqüícolas, duas alternativas são bastante prováveis: a - ou não hidratam seus ovócitos, visto a hidratação ser insuficiente para a flutuação dos ovos;

b - ou os hidratam apenas após a desova, passivamente, evitando dessa maneira os encargos trazidos por uma HPO.

Já para as espécies secundariamente dulceaqüícolas, a estas alternativas acresce-se uma terceira: a de que a HPO ocorra "desnecessariamente", por a espécie ainda não se haver desvinculado deste caráter. Nesta situação, ficaria implícito que ela não atingiu uma completa adaptação ao ambiente de água doce, permitindo supor-se que sua chegada a este meio seja relativamente recente. Tal pode ser o caso, p. ex., de Anchoviella lepidentostole, Clupeidae de migraçöes anádromas que parece hidratar seus ovócitos antes da desova (JUNE FERRAZ DIAS, com. pess.).

Fica proposto, pois, que a ausência de HPO nos ovócitos de teleósteos de água doce derivados de grupos marinhos, trata-se de uma convergência ao comportamento natural apresentado pelos grupos primariamente dulceaqüícolas, apontando como também no nível de gônadas podemos encontrar elementos cuja forma de manifestação é diretamente condicionada pelo meio externo.

\section{AGRADECIMENTOS}

À Dra. Anna Emília A. de M. Vazzoler, pela orientação prestada no trabalho de tese, e às biólogas Mônica Ponz Louro, Maria Rosa Quintans Lopez e Eloci Perez, pelo auxílio nas atividades de coleta e processamento do material.

\section{REFERENCIAS}

AGOSTINHO, A.A. 1985. Estrutura da populaçåo, idade, crescimento e reproduçäo de Rhinelepis aspera (Agassiz, 1829) (Osteich thes, Loricariidae) do rio Paranapanema, PR. Tese de doutorado, Universidade Federal de Såo Carlos, 229p.

AGOSTINHO, A,A; M.Y. NARAHARA, H.M. \& GODINHO, 1982. Morfologia dos ovários de Plecostomus commersonii (Valenciennes, 1840), Osteichthyes-Loricariidae: desenvolvimento dos ovócitos e escala de maturidade. Revta bras. Biol. 42 (1): $71-77$.

ANDREU, B. 1956. Observaciones sobre el ovario de merluza (Merluccius merluccius L.) y características del mecanismo de la puesta. Inv. Pesq. Tomo IV: 49-64.

ANDRIANOV, D.P. \& L.A. LISOVENKO, 1983. New data on the reproduction of the peruvian hake, Merluccius gayi peruanus (Merlucciidae). J. of Icthyology 23(4): $62-72$. 
ARAVIDAN, C.M. \& K.G. PADMANABHAN, 1972. Source of a new crop of oocytes in Tilapia mossambica. Helgolander wiss. Meeresunters 25: 184-192.

BABIKER, M.M. \& H. IBRAHIM, 1979. Studies on the biology of reproduction in the cichlid Tilapia nilotica (L.): gonodal maturation and fecundity. J. Fish Biol. 14 (5): $437-448$.

BARBIERI. M.C.; G. BARBIERI \& M. de A. MARINS, 1981. Sobre a anatomia e histologia de ovários de Geophagus brasiliensis (Quoy \& Gaimard, 1824) na represa do Lobo, estado de São Paulo. Revta bras Biol. 41 (1): 163-168.

BOND, C.E. 1979. Biology of Fishes. Philadelphia, Saunders College Publishing. 514p.

CARAMASCHI, É.P.; H.M. GODINHO \& F. FORESTI, 1982. Reprodução de Hoplias malabaricus (Bloch, 1794) (Teleostei, Erythrinidae) na Represa do rio Pardo (Botucatu, SP). I. Histologia e escala de maturação do ovário. Revta bras. Biol. 42 (3): $635-640$.

CASTILLO, V.R.A. 1986. Estudo sobre a biologia e ciclo de vida de Menticirrhus americanus(Linnaeus, 1758) (Ubatuba 23 30' - Cananéia 25 05'S, S. Paulo). Dissertação de mestrado, Instituto Oceanográfico, Universidade de São Paulo. 150p.

CHAVES, P.T.C. 1985. O desenvolvimento ovocitário em representantes de dez famf́lias de teleó steos a mazônicos: aspectos estruturais e consideraçōes ecológicas. Dissertação de mestrado, Instituto Nacional de Pesquisas da Amazônia / Universidade do Amazonas, Manaus, 83p.

CHAVES, P.T.C. \& A.E.A. de M. VAZZOLER, 1984. Aspectos biologicos de peixes amazônicos: II. Anatomia microscópica de ovários, escala de maturidade e tipo de desova das espécies do gênero Semaprochilodus. Revta bras. Biol. 44 (3): 347-359.

COELLO, S. 1983. Hora de desove de la sardine del sur, Sardinops sagax sagax (Jenyns). Rev. Cienc. Mar. Limm. 2 (1): 239-242.

CRAIK, J.C. \& S.M. HARVEY, 1987. The causes of buoyance in eggs of marine teleostes. J. Mar. Biol. Ass. U.K. 67: 169-182.

CUSSAC, V.E. \& M.C. MAGGESE, 1986. Oogenesis in Rhamdia sapo(Pisces, Pimelodidae). Stages of the oocyte, egg's envelopes and effects of the human chorionic gonadotropin. Revta bras. Biol. 46 (1): 139-147..

DAVIS, T.L.O. 1977. Reproductive biology of the freshwater catfish Tandanus tandanus Mitchell, in the Gwydir River, Australia. I. Structure of the gonads, Aust. J. Mar. Freshwater Res. 28: 139-158.

DEMARTINI, E.E. \& R.K. FOUNTAIN, 1981. Ovarian cycling frequency and batch fecundity in the Queenfish, Seriphus politus:attributes representative of serial spawning fishes. Fishery Bulletin 79 (3): 547-560. 
Vol. 6(3), 1989

ERICKSON, D.L.; M.J. HARRIS, \& G.D. GROSSMAN, 1985. Ovarian cycling of tilefish, Lopholatilus chamaeleonticeps Goode and Bean, from the South Atlantic Bight, USA. J. Fish Biol. 27: 131-385.

FOUCHER, R.P. \& R.J. BEAMISH, 1980. Production of nonviable oocytes by Pacific hake (Merluccius productus).Can. J. Fish. Aquatic Sci. 37: 41-48.

GINZBURG, A.S. 1968. Fertilization in fishes and the problem of polyspermy. Detlaf, T.A. ed. Jerusalem, Israel Program for Scientific Translations. 366p.

GODINHO, H.M.; S. FERRI.; L.O. MEDEIROS, \& J.M.B. BARKER, 1974. Morphological changes in the ovary of Pimelodus maculatus Lacépede, 1803 (Pisces, Siluroidei) related to the reproductive cycle. Revta bras. Biol. 34 (4): 581-588.

HAIMOVICI, M. 1982. Estructura y dinamica poblacional del pargo blanco, Umbrina canosai(Sciaenidae, Pisces) del litoral de Rio Grande do Sul, Brasil, Tese doutoramento, Universidade Nacional de Buenos Aires. 274p.

HAY, D.E.; D.N. OUTRAM ; B.A. McKEOWN, \& M. HURLBURT, 1987. Ovarian development and oocyte diameter as maturation criteria in Pacific herring (Clupea harengus pallasi). Can J. Fish. Aquat. Sci., 44: 1496-1502.

ISAAC-NAHUM, V.J.; A.E.A. de M. VAZZOLER, \& E.M. ZANETI-PRADO, 1983. Estudos sobre estrutura, ciclo de vida e comportamento de Sardinella brasiliensis (Steidachner, 1979) na área entre $22^{\circ} \mathrm{S}$ e $28^{\circ} \mathrm{S}$, Brasil. 3. Morfologia e histologia de ovários e escala de maturidade. Bolm Inst. Oceanogr., S. Paulo 32 (1): 1-16.

KULIKOVA, N.I. \& N. YE. LOSHAKOVA, 1982. Oogenesis and sexual cycle of the long-finned mullet, Liza aurata (Mugilidae). J. of Icthyology 22 (5): 65-75.

MACER, C.T. 1974. The reproductive biology of the horse mackerel Trachurus trachurus (L.) in the North Sea and English Channel. J. Fish Biol. 6: 415-438.

MARCUS, O. \& K. KUSEMIJU, 1984. Some aspects of the reproductive biology of the clupeid Ilisha africana (Bloch) of the Lagos Coast, Nigeria. J. Fish Biol. 25: $679-689$.

MATSUYAMA, M॰; S. MATSUURA.; Y. OUCHI, \& T. HIDAKA, 1987. Maturity classification and group maturity of the red sea bream Pagrus major. I. Female maturity. Marine Biology 96: 163-168.

PARRISH, R.H.; D.L. MALLICOATE, \& R.A. KLINGBELL, 1986. Age dependent fecundity, number of spawnings per year, sex ratio, and maturation stages in northern anchovy, Engraulis mordax. Fishery Bulletin, 84 (3): 503-518.

ROBB, A.P. 1982. Histological observations on the reproductive biology of the haddock, Melanogrammus aeglefinus(L.). J. Fish Biol. 20: 397-408. 
TORRES-VILLEGAS, J.R.; C.G.G. MELGAR ; R॰I. OCHOA-BÅEZ, \& V.A.L. PÉREZ, 1985. Parametros reproductivos de las poblaciones de Opisthonema libertaté(Günther) y discussion sobre su evaluacion por produccion de huevos, Inv. Mar. CICIMAR, 2 (2).

ZANIBONI Fং, E. 1985. Biologia da reproduçāo do matrinxã Brycon cephalus(Günther, 1869) (Teleostei, Characidae). Dissertaçāo de mestrado, Instituto Nacional de Pesquisas da Amazônia / Universidade do Amazonas, Manaus. 134p. 